Supplementary Information to

\title{
Laser Measurements of the H Atom + Ozone Rate Constant at Mesospheric Temperatures
}

\author{
Yingdi Liu, Jian Peng ${ }^{1}$, Kelsey Reppert ${ }^{2}$, Sara Callahan ${ }^{3}$ and Gregory P. Smith*
}

SRI International, 333 Ravenswood Ave, Menlo Park, CA 94025

Current addresses:

1. Coherent Inc., Mountain View CA

2. North Carolina State University, Raleigh NC

3. Smith College, Northampton MA

*Email: gregory.smith@sri.com 
Table S1. Reactions and rate constants used in kinetics simulations and model correction. Rate constant $\mathrm{k}=\operatorname{Aexp}(-\mathrm{Ea} / \mathrm{RT}) \mathrm{T}^{\mathrm{m}}$. Rate constants are from NASA-JPL Evaluation unless otherwise noted, or for $\mathrm{OHv}$ vibrational relaxation.

\begin{tabular}{|c|c|c|c|c|c|}
\hline$\#$ & Reaction & $\begin{array}{c}\text { A-Factor } \\
\left(\mathrm{cm}^{3} \text { molecule }^{-1} \mathrm{~s}^{-1}\right)\end{array}$ & $\mathrm{m}$ & $\begin{array}{c}\mathrm{Ea} \\
\text { (cal/mole) }\end{array}$ & $\begin{array}{c}\text { Source \& } \\
\text { Comments }\end{array}$ \\
\hline 1 & $\mathrm{O}+\mathrm{O}+\mathrm{O}_{2}=>\mathrm{O}_{2}+\mathrm{O}_{2}$ & $2.76 \mathrm{E}-34$ & 0 & -1430 & Baulch76 $\mathrm{N}_{2}$ \\
\hline 2 & $\mathrm{O}+\mathrm{O}+\mathrm{M}=>\mathrm{O}_{2}+\mathrm{M}$ & $5.20 \mathrm{E}-35$ & 0 & -1790 & Baulch76 Ar \\
\hline 3 & $\mathrm{O}+\mathrm{O}_{2}+\mathrm{M}=>\mathrm{O}_{3}+\mathrm{M}$ & $5.29 \mathrm{E}-28$ & -2.4 & 0 & \\
\hline 4 & $\mathrm{O}+\mathrm{O}_{3}=>\mathrm{O}_{2}+\mathrm{O}_{2}$ & $8.00 \mathrm{E}-12$ & 0 & 4094 & \\
\hline 5 & $\mathrm{OD}+\mathrm{CO}_{2}=>\mathrm{O}+\mathrm{CO}_{2}$ & $7.50 \mathrm{E}-11$ & 0 & -229 & \\
\hline 6 & $\mathrm{OD}+\mathrm{Ar}=>\mathrm{O}+\mathrm{Ar}$ & $7.10 \mathrm{E}-13$ & 0 & 0 & \\
\hline 7 & $\mathrm{OD}+\mathrm{O}_{2}=>\mathrm{O}+\mathrm{O}_{2} \mathrm{~S}$ & $3.30 \mathrm{E}-11$ & 0 & -109 & \\
\hline 8 & $\mathrm{OD}+\mathrm{O}_{3}=>\mathrm{O}_{2}+\mathrm{O}_{2}$ & $1.20 \mathrm{E}-10$ & 0 & 0 & \\
\hline 9 & $\mathrm{OD}+\mathrm{O}_{3}=>\mathrm{O}_{2}+\mathrm{O}+\mathrm{O}$ & $1.20 \mathrm{E}-10$ & 0 & 0 & \\
\hline 10 & $\mathrm{OD}+\mathrm{H}_{2} \mathrm{O}_{2}=>\mathrm{OH}+\mathrm{HO}_{2}$ & $5.20 \mathrm{E}-10$ & 0 & 0 & FH76 \\
\hline 11 & $\mathrm{O}+\mathrm{H}_{2} \mathrm{O}_{2}=>\mathrm{OH}+\mathrm{HO}_{2}$ & $1.40 \mathrm{E}-12$ & 0 & 3974 & \\
\hline 12 & $\mathrm{O}+\mathrm{HO}_{2}=>\mathrm{OH}+\mathrm{O}_{2}$ & $3.00 \mathrm{E}-11$ & 0 & -397 & \\
\hline 13 & $\mathrm{O}+\mathrm{OH}=>\mathrm{H}+\mathrm{O}_{2}$ & $1.80 \mathrm{E}-11$ & 0 & -358 & \\
\hline 14 & $\mathrm{O}+\mathrm{OH} 9=>\mathrm{H}+\mathrm{O}_{2}$ & $1.80 \mathrm{E}-11$ & 0 & -358 & reaction $=\mathrm{k}(\mathrm{v}=0)$ \\
\hline 15 & $\mathrm{O}+\mathrm{OH} 8=>\mathrm{H}+\mathrm{O}_{2}$ & $1.80 \mathrm{E}-11$ & 0 & -358 & \\
\hline 16 & $\mathrm{O}+\mathrm{OH} 7=>\mathrm{H}+\mathrm{O}_{2}$ & $1.80 \mathrm{E}-11$ & 0 & -358 & \\
\hline 17 & $\mathrm{O}+\mathrm{OH} 6=>\mathrm{H}+\mathrm{O}_{2}$ & $1.80 \mathrm{E}-11$ & 0 & -358 & \\
\hline 18 & $\mathrm{O}+\mathrm{OH} 5=>\mathrm{H}+\mathrm{O}_{2}$ & $1.80 \mathrm{E}-11$ & 0 & -358 & \\
\hline 19 & $\mathrm{O}+\mathrm{OH} 4=>\mathrm{H}+\mathrm{O}_{2}$ & $1.80 \mathrm{E}-11$ & 0 & -358 & \\
\hline 20 & $\mathrm{O}+\mathrm{OH} 3=>\mathrm{H}+\mathrm{O}_{2}$ & $1.80 \mathrm{E}-11$ & 0 & -358 & \\
\hline 21 & $\mathrm{O}+\mathrm{OH} 2=>\mathrm{H}+\mathrm{O}_{2}$ & $1.80 \mathrm{E}-11$ & 0 & -358 & \\
\hline 22 & $\mathrm{O}+\mathrm{OH} 1=>\mathrm{H}+\mathrm{O}_{2}$ & $1.80 \mathrm{E}-11$ & 0 & -358 & \\
\hline 23 & $\mathrm{H}+\mathrm{O}_{2}(+\mathrm{M})=>\mathrm{HO}_{2}(+\mathrm{M})$ & $5.02 \mathrm{E}-14$ & -0.2 & 0 & \\
\hline 24 & $\mathrm{OH}+\mathrm{O}_{3}=>\mathrm{HO}_{2}+\mathrm{O}_{2}$ & $1.70 \mathrm{E}-12$ & 0 & 1868 & \\
\hline 25 & $\mathrm{HO}_{2}+\mathrm{O}_{3}=>\mathrm{OH}+{ }_{2} \mathrm{O}_{2}$ & $1.00 \mathrm{E}-14$ & 0 & 974 & \\
\hline 26 & $\mathrm{HO}_{2}+\mathrm{HO}_{2}+\mathrm{M}=>\mathrm{H}_{2} \mathrm{O}_{2}+\mathrm{O}_{2}+\mathrm{M}$ & $2.10 \mathrm{E}-33$ & 0 & -1828 & \\
\hline 27 & $\mathrm{OH}+\mathrm{HO}_{2}=>\mathrm{H}_{2} \mathrm{O}+\mathrm{O}_{2}$ & 4.80E-11 & 0 & -497 & \\
\hline 28 & $\mathrm{HO}_{2}+\mathrm{HO}_{2}=>\mathrm{H}_{2} \mathrm{O}_{2}+\mathrm{O}_{2}$ & $3.00 \mathrm{E}-13$ & 0 & -914 & \\
\hline 29 & $\mathrm{OH}+\mathrm{H}_{2} \mathrm{O}_{2}=>\mathrm{HO}_{2}+\mathrm{H}_{2} \mathrm{O}$ & $1.80 \mathrm{E}-12$ & 0 & 0 & \\
\hline 30 & $\mathrm{OH}+\mathrm{OH}=>\mathrm{O}+\mathrm{H}_{2} \mathrm{O}$ & $1.80 \mathrm{E}-12$ & 0 & 0 & \\
\hline
\end{tabular}




\begin{tabular}{|c|c|c|c|c|c|}
\hline 31 & $\mathrm{H}+\mathrm{HO}_{2}=>\mathrm{OH}+\mathrm{OH}$ & $7.20 \mathrm{E}-11$ & 0 & 0 & \\
\hline 32 & $\mathrm{H}+\mathrm{HO}_{2}=>\mathrm{O}+\mathrm{H}_{2} \mathrm{O}$ & $1.60 \mathrm{E}-12$ & 0 & 0 & \\
\hline 33 & $\mathrm{H}+\mathrm{HO}_{2}=>\mathrm{H}_{2}+\mathrm{O}_{2}$ & $6.90 \mathrm{E}-12$ & 0 & 0 & \\
\hline 34 & $\mathrm{OH}+\mathrm{OH}=>\mathrm{H}_{2} \mathrm{O}_{2}$ & $2.60 \mathrm{E}-11$ & 0 & 0 & \\
\hline 35 & $\mathrm{H}+\mathrm{OH}+\mathrm{M}=>\mathrm{H}_{2} \mathrm{O}+\mathrm{M}$ & $6.10 \mathrm{E}-26$ & -2 & 0 & \\
\hline 36 & $\mathrm{H}+\mathrm{H}_{2} \mathrm{O}_{2}=>\mathrm{HO}_{2}+\mathrm{H}_{2}$ & $2.80 \mathrm{E}-12$ & 0 & 3756 & \\
\hline 37 & $\mathrm{H}+\mathrm{H}_{2} \mathrm{O}_{2}=>\mathrm{OH}+\mathrm{H}_{2} \mathrm{O}$ & $1.70 \mathrm{E}-11$ & 0 & 3577 & \\
\hline 38 & $\mathrm{OD}+\mathrm{H}_{2} \mathrm{O}=>\mathrm{OH}+\mathrm{OH}$ & $1.11 \mathrm{E}-10$ & 0 & -119 & \\
\hline 39 & $\mathrm{OD}+\mathrm{H}_{2} \mathrm{O}=>\mathrm{OH} 1+\mathrm{OH} 1$ & $3.70 \mathrm{E}-11$ & 0 & -119 & \\
\hline 40 & $\mathrm{OD}+\mathrm{H}_{2} \mathrm{O}=>\mathrm{OH}_{2}+\mathrm{OH}_{2}$ & $1.50 \mathrm{E}-11$ & 0 & -119 & \\
\hline 41 & $\mathrm{OH} 9+\mathrm{O}_{2}=>\mathrm{OH} 8+\mathrm{O}_{2}$ & $2.20 \mathrm{E}-11$ & 0 & 0 & $\mathrm{KSC} 11$ \\
\hline 42 & $\mathrm{OH} 8+\mathrm{O}_{2}=>\mathrm{OH} 7+\mathrm{O}_{2}$ & $1.00 \mathrm{E}-11$ & 0 & 0 & interpolate \\
\hline 43 & $\mathrm{OH} 7+\mathrm{O}_{2}=>\mathrm{OH} 6+\mathrm{O}_{2}$ & $7.00 \mathrm{E}-12$ & 0 & 0 & “ \\
\hline 44 & $\mathrm{OH} 6+\mathrm{O}_{2}=>\mathrm{OH} 5+\mathrm{O}_{2}$ & $3.00 \mathrm{E}-12$ & 0 & 0 & “ \\
\hline 45 & $\mathrm{OH} 5+\mathrm{O}_{2}=>\mathrm{OH} 4+\mathrm{O}_{2}$ & $1.70 \mathrm{E}-12$ & 0 & 0 & DLB91 \\
\hline 46 & $\mathrm{OH} 4+\mathrm{O}_{2}=>\mathrm{OH}_{3}+\mathrm{O}_{2}$ & $8.80 \mathrm{E}-13$ & 0 & 0 & DLB91 \\
\hline 47 & $\mathrm{OH} 3+\mathrm{O}_{2}=>\mathrm{OH}_{2}+\mathrm{O}_{2}$ & $5.20 \mathrm{E}-13$ & 0 & 0 & DLB91 \\
\hline 48 & $\mathrm{OH} 2+\mathrm{O}_{2}=>\mathrm{OH} 1+\mathrm{O}_{2}$ & $2.70 \mathrm{E}-13$ & 0 & 0 & DLB91 \\
\hline 49 & $\mathrm{OH} 1+\mathrm{O}_{2}=>\mathrm{OH}+\mathrm{O}_{2}$ & $1.30 \mathrm{E}-13$ & 0 & 0 & DLB91 \\
\hline 50 & $\mathrm{OH} 9+\mathrm{H}_{2} \mathrm{O}=>\mathrm{OH} 8+\mathrm{H}_{2} \mathrm{O}$ & $1.00 \mathrm{E}-10$ & 0 & 0 & extrapolate \\
\hline 51 & $\mathrm{OH} 8+\mathrm{H}_{2} \mathrm{O}=>\mathrm{OH} 7+\mathrm{H}_{2} \mathrm{O}$ & $1.00 \mathrm{E}-10$ & 0 & 0 & “ \\
\hline 52 & $\mathrm{OH} 7+\mathrm{H}_{2} \mathrm{O}=>\mathrm{OH} 6+\mathrm{H}_{2} \mathrm{O}$ & $9.00 \mathrm{E}-11$ & 0 & 0 & “ \\
\hline 53 & $\mathrm{OH} 6+\mathrm{H}_{2} \mathrm{O}=>\mathrm{OH} 5+\mathrm{H}_{2} \mathrm{O}$ & $8.00 \mathrm{E}-11$ & 0 & 0 & “ \\
\hline 54 & $\mathrm{OH} 5+\mathrm{H}_{2} \mathrm{O}=>\mathrm{OH} 4+\mathrm{H}_{2} \mathrm{O}$ & $6.50 \mathrm{E}-11$ & 0 & 0 & “ \\
\hline 55 & $\mathrm{OH} 4+\mathrm{H}_{2} \mathrm{O}=>\mathrm{OH}_{3}+\mathrm{H}_{2} \mathrm{O}$ & $5.00 \mathrm{E}-11$ & 0 & 0 & “ \\
\hline 56 & $\mathrm{OH}_{3}+\mathrm{H}_{2} \mathrm{O}=>\mathrm{OH}_{2}+\mathrm{H}_{2} \mathrm{O}$ & $4.00 \mathrm{E}-11$ & 0 & 0 & “ \\
\hline 57 & $\mathrm{OH}_{2}+\mathrm{H}_{2} \mathrm{O}=>\mathrm{OH} 1+\mathrm{H}_{2} \mathrm{O}$ & $2.58 \mathrm{E}-11$ & 0 & 0 & RJR90 \\
\hline 58 & $\mathrm{OH} 1+\mathrm{H}_{2} \mathrm{O}=>\mathrm{OH}+\mathrm{H}_{2} \mathrm{O}$ & $1.33 \mathrm{E}-11$ & 0 & 0 & RJR90 \\
\hline 59 & $\mathrm{OH} 9+\mathrm{O}_{3}=>\mathrm{OH} 8+\mathrm{O}_{3}$ & $1.40 \mathrm{E}-10$ & 0 & 0 & $\mathrm{KSC} 11$ \\
\hline 60 & $\mathrm{OH} 8+\mathrm{O}_{3}=>\mathrm{OH} 7+\mathrm{O}_{3}$ & $5.00 \mathrm{E}-11$ & 0 & 0 & interpolate \\
\hline 61 & $\mathrm{OH} 7+\mathrm{O}_{3}=>\mathrm{OH} 6+\mathrm{O}_{3}$ & $4.00 \mathrm{E}-11$ & 0 & 0 & “ \\
\hline 62 & $\mathrm{OH} 6+\mathrm{O}_{3}=>\mathrm{OH} 5+\mathrm{O}_{3}$ & $3.00 \mathrm{E}-11$ & 0 & 0 & “ \\
\hline 63 & $\mathrm{OH} 5+\mathrm{O}_{3}=>\mathrm{OH} 4+\mathrm{O}_{3}$ & $2.00 \mathrm{E}-11$ & 0 & 0 & “ \\
\hline 64 & $\mathrm{OH} 4+\mathrm{O}_{3}=>\mathrm{OH}_{3}+\mathrm{O}_{3}$ & $1.36 \mathrm{E}-11$ & 0 & 0 & TAS88 \\
\hline 65 & $\mathrm{OH} 3+\mathrm{O}_{3}=>\mathrm{OH}_{2}+\mathrm{O}_{3}$ & $6.80 \mathrm{E}-12$ & 0 & 0 & TAS88 \\
\hline 66 & $\mathrm{OH} 2+\mathrm{O}_{3}=>\mathrm{OH} 1+\mathrm{O}_{3}$ & $3.00 \mathrm{E}-12$ & 0 & 0 & TAS88 \\
\hline 67 & $\mathrm{OH} 1+\mathrm{O}_{3}=>\mathrm{OH}+\mathrm{O}_{3}$ & $1.00 \mathrm{E}-12$ & 0 & 0 & TAS88 \\
\hline 68 & $\mathrm{OH} 9+\mathrm{O}=>\mathrm{OH} 8+\mathrm{O}$ & $3.67 \mathrm{E}-10$ & 0 & 0 & KSC11(-reaction) \\
\hline 69 & $\mathrm{OH} 8+\mathrm{O}=>\mathrm{OH} 7+\mathrm{O}$ & $2.20 \mathrm{E}-10$ & 0 & -238 & cascade model; \\
\hline
\end{tabular}




\begin{tabular}{|c|c|c|c|c|c|}
\hline 70 & $\mathrm{OH} 7+\mathrm{O}=>\mathrm{OH} 6+\mathrm{O}$ & $1.70 \mathrm{E}-10$ & 0 & -238 & interpolate \\
\hline 71 & $\mathrm{OH} 6+\mathrm{O}=>\mathrm{OH} 5+\mathrm{O}$ & $1.20 \mathrm{E}-10$ & 0 & -238 & “ \\
\hline 72 & $\mathrm{OH} 5+\mathrm{O}=>\mathrm{OH} 4+\mathrm{O}$ & $8.00 \mathrm{E}-11$ & 0 & -238 & “ \\
\hline 73 & $\mathrm{OH} 4+\mathrm{O}=>\mathrm{OH}_{3}+\mathrm{O}$ & $5.50 \mathrm{E}-11$ & 0 & -238 & SCR15(-reaction) \\
\hline 74 & $\mathrm{OH} 3+\mathrm{O}=>\mathrm{OH}_{2}+\mathrm{O}$ & $2.70 \mathrm{E}-11$ & 0 & -238 & $\mathrm{BMC02}$ \\
\hline 75 & $\mathrm{OH} 2+\mathrm{O}=>\mathrm{OH} 1+\mathrm{O}$ & $1.07 \mathrm{E}-11$ & 0 & -238 & ВMC02 \\
\hline 76 & $\mathrm{OH} 1+\mathrm{O}=>\mathrm{OH}+\mathrm{O}$ & $5.35 \mathrm{E}-12$ & 0 & -238 & extrapolate \\
\hline 77 & $\mathrm{H}+\mathrm{O}_{3}=>\mathrm{OH} 9+\mathrm{O}_{2}$ & $6.22 \mathrm{E}-11$ & 0 & 934 & branch A-G97 \\
\hline 78 & $\mathrm{H}+\mathrm{O}_{3}=>\mathrm{OH} 8+\mathrm{O}_{2}$ & $3.86 \mathrm{E}-11$ & 0 & 934 & “ \\
\hline 79 & $\mathrm{H}+\mathrm{O}_{3}=>\mathrm{OH} 7+\mathrm{O}_{2}$ & 3.09E-11 & 0 & 934 & “ \\
\hline 80 & $\mathrm{H}+\mathrm{O}_{3}=>\mathrm{OH} 6+\mathrm{O}_{2}$ & $6.20 \mathrm{E}-12$ & 0 & 934 & “ \\
\hline 81 & $\mathrm{H}+\mathrm{O}_{3}=>\mathrm{OH} 5+\mathrm{O}_{2}$ & $2.00 \mathrm{E}-12$ & 0 & 934 & “ \\
\hline 82 & $\mathrm{OD}+\mathrm{H}_{2}=>\mathrm{OH}+\mathrm{H}$ & $1.85 \mathrm{E}-11$ & 0 & 0 & branch AS86 \\
\hline 83 & $\mathrm{OD}+\mathrm{H}_{2}=>\mathrm{OH} 1+\mathrm{H}$ & $2.95 \mathrm{E}-11$ & 0 & 0 & “ \\
\hline 84 & $\mathrm{OD}+\mathrm{H}_{2}=>\mathrm{OH}_{2}+\mathrm{H}$ & $3.30 \mathrm{E}-11$ & 0 & 0 & “ \\
\hline 85 & $\mathrm{OD}+\mathrm{H}_{2}=>\mathrm{OH}_{3}+\mathrm{H}$ & $2.50 \mathrm{E}-11$ & 0 & 0 & “ \\
\hline 86 & $\mathrm{OD}+\mathrm{H}_{2}=>\mathrm{OH} 4+\mathrm{H}$ & $1.40 \mathrm{E}-11$ & 0 & 0 & “ \\
\hline 87 & $\mathrm{O}+\mathrm{H}_{2}=>\mathrm{OH}+\mathrm{H}$ & $8.50 \mathrm{E}-20$ & 2.67 & 6286 & \\
\hline 88 & $\mathrm{OH}+\mathrm{H}_{2}=>\mathrm{H}+\mathrm{H}_{2} \mathrm{O}$ & $2.80 \mathrm{E}-12$ & 0 & 3577 & \\
\hline 89 & $\mathrm{OH} 9+\mathrm{AR}=>\mathrm{OH} 8+\mathrm{Ar}$ & $2.00 \mathrm{E}-14$ & 0 & 0 & est. \\
\hline 90 & $\mathrm{OH} 9+\mathrm{H}=>\mathrm{OH} 8+\mathrm{H}$ & $4.00 \mathrm{E}-10$ & 0 & 0 & est. same as $\mathrm{O}$ \\
\hline 91 & $\mathrm{OH} 9+\mathrm{OH}=>\mathrm{OH} 8+\mathrm{OH}$ & $4.00 \mathrm{E}-10$ & 0 & 0 & est. same as $\mathrm{O}$ \\
\hline
\end{tabular}

\section{References:}

A-G97: S. Adler-Golden, "Kinetic parameters for $\mathrm{OH}$ nightglow modeling consistent with recent laboratory measurements,” J. Geophys. Res. 102, 19969-19976 (1997).

NASA-JPL11: S. P. Sander, R. R. Friedl, J. R. Barker, D. M. Golden, M. J. Kurylo, P. H. Wine, J. P. D. Abbatt, J. B. Burkholder, C. E. Kolb, G. K. Moortgat, R. E. Huie, V. L. Orkin, "Chemical kinetics and photochemical data for use in stratospheric modeling, Evaluation Number 17," National Aeronautics and Space Administration, jpldataeval.jpl.gov, Jet Propulsion Laboratory, 2011. 
FH76: I. S. Fletcher, D. Husain, "The collisional quenching of electronically excited oxygen atoms by the gases $\mathrm{NH} 3, \mathrm{H} 2 \mathrm{O} 2, \mathrm{C} 2 \mathrm{H} 6, \mathrm{C} 3 \mathrm{H} 8$, and $\mathrm{C}(\mathrm{CH} 3) 4$ using time-resiolved attenuation of atomic resonance radiation,” Can. J. Chem. 54, 1765 (1976).

Baulch76: D. L. Baulch, D. D. Drysdale, J. Duxbury, S. Grant, "Evaluated Kinetic Data for High Temperature Reactions," vol. 3, Butterworths, London, 1976.

BMC02: J. E. Boulter, J. Marschall, and R. A. Copeland, "Measurement of $\mathrm{OH}(\mathrm{v}=2,3,4)$ collisional removal rate constants by O,” EOS 83, S231 (2002).

SCR15: G. P. Smith, R. A. Copeland, R. Robertson, “Temperature dependent rate constants for $\mathrm{OH}(\mathrm{v}=4)+\mathrm{O}, "$ to be submitted, 2015

AS86: P. M. Aker, J. J. Sloan, The initial product vibrational energy distribution in the reaction between $\mathrm{O}\left({ }^{1} \mathrm{D}_{2}\right)$ and $\mathrm{H}_{2}$, , J. Chem. Phys. 85, 1412 (1986).

KSC11: K. S. Kalogerakis, G. P. Smith, and R. A. Copeland, "Collisional Removal of OH(X²P, v=9) by $\mathrm{O}, \mathrm{O}_{2}, \mathrm{O}_{3}, \mathrm{~N}_{2}$, and $\mathrm{CO}_{2}$," J. Geophys. Res. 116, D20307 (2011).

TAS88: H. Teitelbaum, P. Aker, J. J. Sloan, “An experimentally determined set of V-T and V-V rate constants involving the $\mathrm{OH}$ radical. Implications for atmospheric chemistry,” Chem. Phys. 119, 79-87 (1988).

DLB91: J. A. Dodd, S. J. Lipson and A. M. Blumberg, "Formation and vibrational relaxation of $\mathrm{OH}\left(\mathrm{X}^{2} \mathrm{P}_{\mathrm{i}}, \mathrm{v}\right)$ by $\mathrm{O}_{2}$ and $\mathrm{CO}_{2}$, ,J. Chem. Phys. 95, 5752 (1991).

RJR90: G. A. Raiche, J. B. Jeffries, K. J. Rensberger, D. R. Crosley, "Vibrational energy transfer in $\mathrm{OH}\left(\mathrm{X}^{2} \mathrm{P}_{\mathrm{i}}\right), \mathrm{v}=2$ and 1," J. Chem. Phys. 92, 7258 (1990). 